\title{
A retrospective study of postoperative radiotherapy for locally advanced esophageal squamous cell carcinoma
}

\author{
Zhaoyue Zhang ${ }^{1,2 \#}$, Liping X ${ }^{1,2 \#}$, Xiaoke $\mathrm{Di}^{1}$, Chi Zhang ${ }^{1}$, Xiaolin $\mathrm{Ge}^{1}$, Xinchen Sun ${ }^{1}$ \\ ${ }^{1}$ Department of Radiation Therapy, Nanjing Medical University First Affiliated Hospital, Nanjing, China; ${ }^{2}$ The First Clinical College, Nanjing \\ Medical University, Nanjing, China \\ Contributions: (I) Conception and design: X Ge, X Sun; (II) Administrative support: X Sun; (III) Provision of study materials or patients: X Ge, X Sun; \\ (IV) Collection and assembly of data: Z Zhang, L Xu, X Di, C Zhang, X Ge; (V) Data analysis and interpretation: Z Zhang, L Xu, X Di, C Zhang, \\ X Ge; (VI) Manuscript writing: All authors; (VII) Final approval of manuscript: All authors. \\ \#These authors contributed equally to this work. \\ Correspondence to: Xinchen Sun; Xiaolin Ge. Department of Radiation Therapy, Nanjing Medical University First Affiliated Hospital, \\ No. 300 Guangzhou Road, Nanjing, China. Email: sunxinchen2012@163.com; doctorgx1@163.com.
}

Background: The optimal therapeutic strategy in locally advanced esophageal squamous cell carcinoma (ESCC) primarily treated by surgery remains unknown. This study was designed to evaluate the impact of postoperative chemoradiotherapy and postoperative sequential chemoradiotherapy on survival in this population.

Methods: The study included a total of 228 consecutive patients who underwent radical esophagectomy and were confirmed to have stage pT3-4 or pN+ ESCC from September 2011 to September 2017 at our institution. All patients received postoperative radiotherapy with or without concurrent or sequential chemotherapy after esophagectomy. Univariate and multivariate analyses were used to compare the survival of patients with postoperative radiotherapy, postoperative concurrent chemoradiotherapy, and postoperative sequential chemoradiotherapy.

Results: After a median follow-up of 52 months, the 3- and 5-year overall survival (OS) rates were $70.2 \%$ [95\% confidence interval (CI), 63.7-76.7\%] and 62.2\% (95\% CI, 54.6-69.8\%), respectively. The disease-free survival (DFS) rates at 3 and 5 years were $65.2 \%$ (95\% CI, 58.7-71.7\%) and 55.2\% (95\% CI, 47.6-62.8\%), respectively. The 3- and 5-year locoregional recurrence-free survival (LRFS) rates were 65.1\% (95\% CI, $58.4-71.8 \%$ ) and $55.5 \%$ (95\% CI, 47.7-63.3\%). Of the 228 patients, 38 (16.7\%) had distant metastases. Subgroup analysis showed that being male and having a higher $\mathrm{T}$ stage were independent poor prognostic factors for OS and DFS in patients with pN+ or stage III + IVA ESCC. The results also showed that in patients with stage III + IVA ESCC, the DFS of the patients in the concurrent chemotherapy (CCT) group was improved compared with that in the no CCT group [hazard ratio (HR), 0.551; 95\% CI, 0.323-0.938; $\mathrm{P}=0.028]$. Multivariate analysis showed that sequential chemoradiotherapy was associated with poor LRFS (HR, 2.312; 95\% CI, 1.078-4.959; $\mathrm{P}=0.031$ ), especially in stage T3-4 patients, and it was also related to the poor DFS (HR, 1.781; 95\% CI, 1.086-2.921; $\mathrm{P}=0.022$ ) in patients with stage T3-4 ESCC.

Conclusions: In patients with locally advanced ESCC, those who underwent sequential chemoradiotherapy had a worse LRFS. Postoperative concurrent chemoradiotherapy was the most effective adjuvant therapy for resected stage III-IVA ESCC. In addition, being male, having a higher T stage, and being node-positive were independent poor prognostic factors for OS and DFS.

Keywords: Esophageal squamous cell carcinoma (ESCC); esophagectomy; postoperative chemoradiotherapy; postoperative sequential chemoradiotherapy; prognosis

Submitted Sep 28, 2019. Accepted for publication Nov 05, 2019.

doi: 10.21037/apm.2019.11.19

View this article at: http://dx.doi.org/10.21037/apm.2019.11.19 


\section{Introduction}

Esophageal cancer is predicted to be the seventh most commonly diagnosed cancer and the sixth leading cause of cancer death worldwide in 2018, with about 57,200 new cases and 50,900 deaths annually (1). It ranks fifth in incidence and fourth in mortality in China (2). Surgery is a major component of treatment for esophageal cancer, but the 5 -year relative survival rate is still only $19 \%$, with a rate of $45 \%$ for localized tumors and $24 \%$ for regional tumors (3). Therefore, the survival benefit from surgery is limited, which provides a basis for comprehensive treatment. Although the National Comprehensive Cancer Network (NCCN) guidelines do not recommend further adjuvant therapy for patients with esophageal squamous cell carcinoma (ESCC) after R0 resection (4), postoperative radiotherapy was shown to improve overall survival (OS) especially for patients with stage III ESCC or positive lymph nodes $(5,6)$. We want to further support this conclusion through our retrospective study to see if postoperative chemoradiotherapy or sequential chemoradiotherapy is effective for ESCC.

\section{Methods}

\section{Patients}

From September 2011 to September 2017, patients diagnosed with stage pT3-4 and $\mathrm{pN}+\mathrm{ESCC}$ who were definitively treated with postoperative radiation, with or without concurrent or sequential chemotherapy after esophagectomy, were selected for this study. Patients who did not complete postoperative radiotherapy, had a history of another malignancy, received preoperative chemotherapy, or were in stage M1 were excluded.

Tumor location was determined by the results of preoperative esophagoscopy, while tumor size, extent of nodal disease, tumor differentiation, lymphovascular invasion (LVI), and nerve invasion were based on pathology reports. The eighth edition of the American Joint Committee on Cancer staging system was used to determine the pathologic stage of the disease.

\section{Postoperative radiotherapy}

Radiotherapy was administered within 2 months of surgery using intensity-modulated radiation therapy (IMRT). The clinical tumor volume (CTV) included the lower cervical region, whole esophageal region (for upper esophageal cancer), supraclavicular area, 1, 2, 3p, 4, 7, and 8 (middle and lower) nodal regions, and celiac trunk region (lower). The planning target volume (PTV) included the CTV plus an expansion margin of $0.5 \mathrm{~cm}$.

A total dose of 45 to 50 Gy in 25 fractions was administered within 5 weeks. To reduce the incidence of postoperative pulmonary complications, the total proportion of the lungs receiving 5 Gy was limited to $50 \%$, the total proportion of the lungs receiving $20 \mathrm{~Gy}$ was limited to $20 \%$, and the mean dose was set at $16 \mathrm{~Gy}$. The dose to the heart was also limited, with the proportion of the heart receiving 30 Gy kept to $30 \%$ and below $\left(\mathrm{V}_{30 \mathrm{~Gy}}\right.$ $\leq 30 \%)$.

\section{Chemotherapy}

After surgery, patients underwent either sequential chemotherapy, concurrent chemotherapy (CCT), or both. According to the clinicians' discretion, the patient's status, and the personal wishes of the patient, the chemotherapy regimens administered in this study were not completely the same. Chemotherapy regimens included the following: $\mathrm{S}-1$ at $40 \mathrm{mg}\left(<1.4 \mathrm{~m}^{2}\right) / 50 \mathrm{mg}\left(>1.4 \mathrm{~m}^{2}\right.$ and $\left.<1.6 \mathrm{~m}^{2}\right) / 60 \mathrm{mg}$ $\left(>1.6 \mathrm{~m}^{2}\right)$ orally twice a day on days $1-28$, cycled every 42 days for 1 cycle (for concurrent chemoradiotherapy) or 4-6 cycles (for sequential chemoradiotherapy); fluorouracil at $450 \mathrm{mg} / \mathrm{m}^{2}$ by continuous intravenous (IV) infusion daily on days $1-5+$ cisplatin at $75 \mathrm{mg} / \mathrm{m}^{2}$ by $\mathrm{IV}$ on day 1 , cycled every 21 days for 1-2 cycles (for concurrent chemoradiotherapy) or 4-6 cycles (for sequential chemoradiotherapy); and docetaxel at $75 \mathrm{mg} / \mathrm{m}^{2}$ by IV on day $1+$ cisplatin at $75 \mathrm{mg} / \mathrm{m}^{2}$ by IV on day 2 , cycled every 21 days for $1-2$ cycles (for concurrent chemoradiotherapy) or 4-6 cycles (for sequential chemoradiotherapy).

\section{Follow-up}

Patients were instructed to return periodically for follow-up evaluations every 3 months for the first year, every 6 months for the next 2 years, and annually thereafter. The survival status for patients who did not come at the scheduled follow-up times was updated by means of telephone calls every 6 months. OS was measured from the date of surgery to the date of death or last follow-up. Disease-free survival (DFS) was measured from the date of surgery to the date of first evidence of relapse or death as a result of any cause, whichever was observed first. Locoregional recurrence-free survival (LRFS) rate was measured from the date of surgery 
to the date of first evidence of local or regional recurrence. Recurrences or metastases were documented by means of clinical examinations including computed tomography (CT), esophagoscopy plus biopsy, or positron emission tomography $(\mathrm{PET}) / \mathrm{CT}$.

\section{Statistical analysis}

Survival analysis by the Kaplan-Meier method was performed using the SPSS 23.0 statistical software. Multivariate analyses using Cox proportional hazards survival regression model to evaluate the influence of covariates on OS, DFS, and LRFS. Significant values were defined as those with a $\mathrm{P}<0.05$.

\section{Results}

\section{Patient characteristics}

Between September 2011 and November 2017, we enrolled 257 patients in the study, of whom 228 met the inclusion criteria and were eligible to participate. The patient and tumor characteristics are shown in Table 1.

\section{Survival}

After a median follow-up of 52 (range from 16 to 88) months, the median OS and DFS was 34 and 32 months, respectively. The 3- and 5-year OS rates were 70.2\% [95\% confidence interval (CI), 63.7-76.7\%] and 62.2\% (95\% CI, $54.6-69.8 \%$ ), respectively (Figure $1 A$ ). The DFS rates at 3 and 5 years were $65.2 \%$ (95\% CI, 58.7-71.7\%) and $55.2 \%$ (95\% CI, 47.6-62.8\%), respectively (Figure 1B). The 3and 5-year LRFS rates were $65.1 \%$ (95\% CI, 58.4-71.8\%) and $55.5 \%$ (95\% CI, 47.7-63.3\%; Figure 1C). Of the 228 patients, 38 (16.7\%) had distant metastases, including lung, liver, renal, brain, and bone metastases.

\section{Univariate and multivariate analyses}

Table 1 outlines the results of the univariate analysis. For the 94 patients with pathologic stage II disease, the 5-year OS, DFS, and LRFS rates were 75.0\% (95\% CI, 64.4-85.6\%), $70.4 \%$ (95\% CI, $59.8-81.0 \%$ ), and $70.9 \%$ (95\% CI, $60.3-$ $81.5 \%)$. For the remaining 134 patients with pathologic stage III + IVA disease, the 5-year OS, DFS, and LRFS rates were $52.9 \%$ (95\% CI, 42.5-63.3\%), 43.7\% (95\% CI, $33.3-54.1 \%$ ), and $43.6 \%$ (95\% CI, 32.4-54.8\%).
There was significantly improved survival in patients who were female, with stage $\mathrm{N}$ - disease, in stage II, and with no LVI or nerve invasion. Patients with $\mathrm{N}$ - disease, in stage II, or with no nerve invasion had a significantly higher LRFS than those with $\mathrm{N}+$ disease, in stage III, or with nerve invasion.

There was no significant difference in the 5 -year OS, DFS, or LRFS rates between patients with or without CCT. However, subgroup analysis showed that for male patients with $\mathrm{pN}+$ disease, the 5 -year DFS rate $(30.6 \%$ vs. $49.3 \%, \mathrm{P}=0.027)$ and 5 -year LRFS rate $(64.1 \%$ vs. $76.2 \%$, $\mathrm{P}=0.037)$ of the CCT group were better compared with the postoperative radiotherapy group, but there was no significant difference in the 5 -year OS rate $(45.8 \% v s$. $50.7 \%, \mathrm{P}=0.251)$. After CCT, the 5 -year DFS rate $(42.4 \%$ vs. $72.7 \%, \mathrm{P}=0.037)$ was also improved in stage III + IVA patients who were $\leq 60$ years old and the 5 -year OS rate (55.9\% vs. $71.2 \%, \mathrm{P}=0.263)$ and 5 -year LRFS rate $(67.4 \%$ vs. $80.8 \%, \mathrm{P}=0.181)$ seemed to have an increasing trend, but there was no statistically significant difference. The 5 -year OS rate $(68.1 \%$ vs. $51.2 \%, \mathrm{P}=0.035)$, 5-year DFS rate (63.0\% vs. $41.3 \%, \mathrm{P}=0.007)$, and 5 -year LRFS rate $(84.2 \%$ vs. $66.7 \%, \mathrm{P}=0.006)$ were all decreased in the sequential chemotherapy group compared with the postoperative radiotherapy group. When the chemotherapy group was further divided into the no chemotherapy, CCT, sequential chemotherapy, and concurrent + sequential chemotherapy groups, the 5 -year OS $(53.0 \%$ vs. $72.1 \%, \mathrm{P}=0.036)$ and 5 -year DFS (36.5\% vs. $64.5 \%, \mathrm{P}=0.007)$ of the patients with sequential chemotherapy were significantly lower than those without chemotherapy. Although there was no statistically significant difference in the 5 -year LRFS rate $(66.1 \%$ vs. $81.4 \%, \mathrm{P}=0.078)$, a similar trend could still be observed.

Figure 2 shows the significant results of the multivariate analysis of OS, DFS, and LRFS, respectively. The factors that were significantly correlated with poor OS and DFS were being male, having higher T stage, and having positive lymph nodes. The factor that was significantly correlated with poor LRFS was sequential chemotherapy.

Subgroup analysis showed that being male and having a higher $\mathrm{T}$ stage remained independent poor prognostic factors for OS and DFS in patients with $\mathrm{pN}+$ or stage III + IVA disease. The results also showed that patients with CCT had higher DFS than those without CCT in patients with stage III + IVA disease [hazard ratio (HR), 0.551; 95\% CI, 0.323-0.938; $\mathrm{P}=0.028]$. However, the factors mentioned above have no effect on LRFS. For patients with pN0 disease, the LRFS was significantly lower in the concurrent + 
Table 1 Univariate analysis for survival

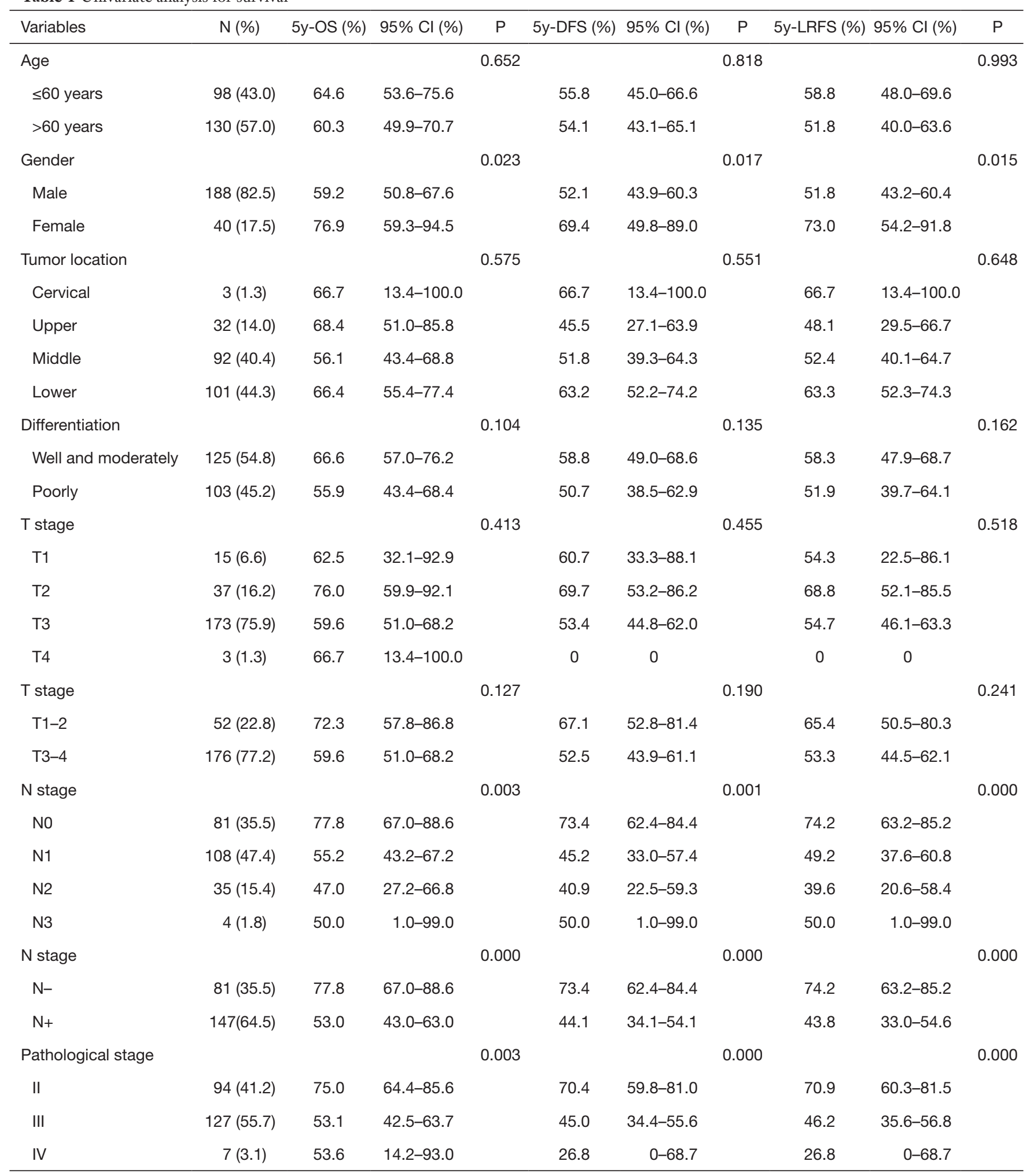

Table 1 (continued) 
Table 1 (continued)

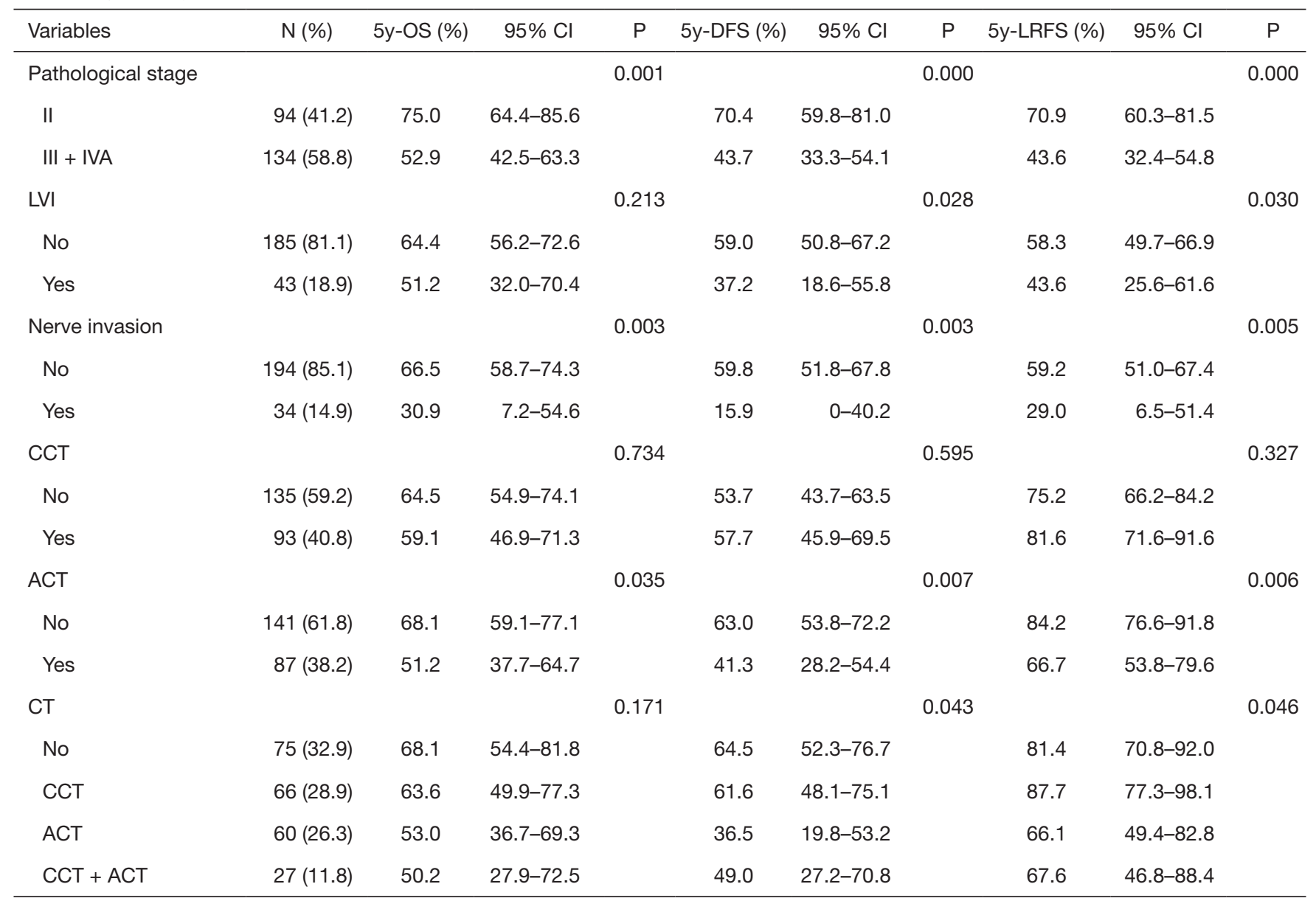

OS, overall survival; DFS, disease-free survival; LRFS, locoregional relapse-free survival; $5 y$, 5 years; Cl, confidence interval; LVI, lymphovascular invasion; CCT, concurrent chemotherapy; ACT, adjuvant chemotherapy; CT, chemotherapy.
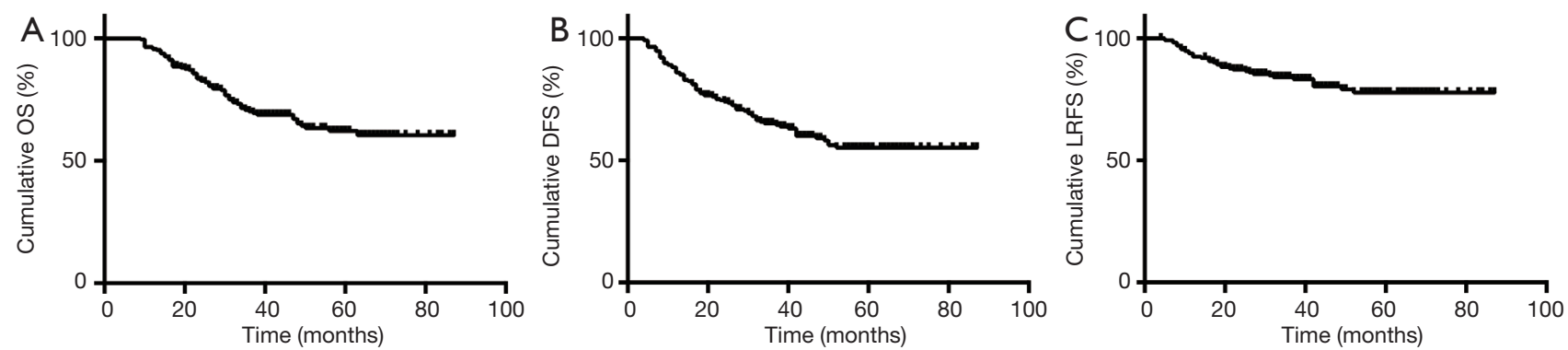

Figure 1 Kaplan-Meier survival curve of the 228 patients administered postoperative radiotherapy. (A) OS; (B) DFS; (C) LRFS. OS, overall survival; DFS, disease-free survival; LRFS, locoregional recurrence-free survival. 

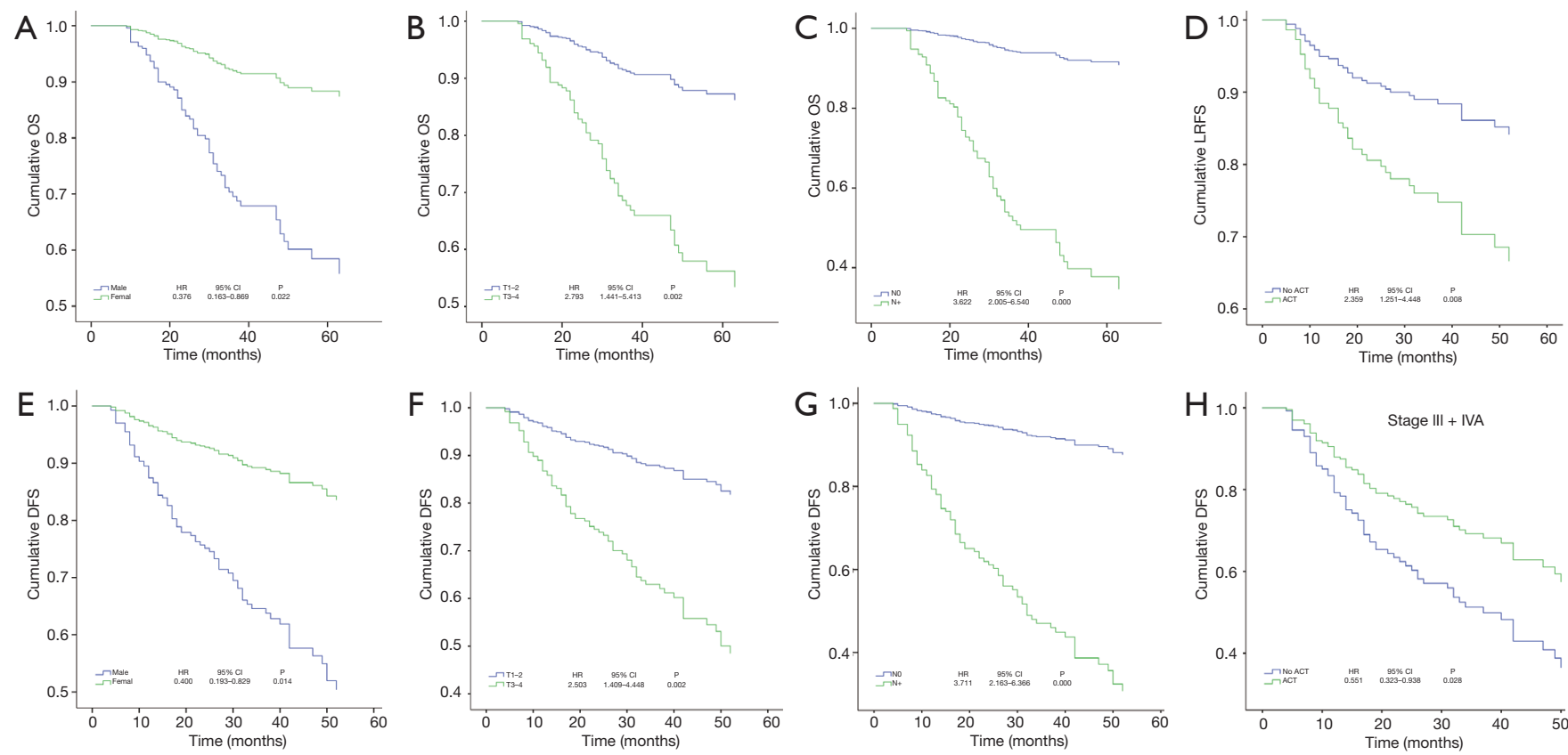

Figure 2 Cox survival curves of OS, DFS, and LRFS for different factors. OS analyzed according to (A) sex, (B) T stage, and (C) N stage. (D) ACT LRFS; DFS analyzed according to (E) sex, (F) T stage, and (G) N stage. (H) CCT DFS for stage III + IVA patients. OS, overall survival; DFS, disease-free survival; LRFS, locoregional relapse-free survival; ACT, adjuvant chemotherapy; CCT, concurrent chemotherapy.

sequential chemotherapy group compared with patients who did not receive any form of chemotherapy (HR, 8.666; 95\% CI, 1.930-38.920; $\mathrm{P}=0.005)$. In T3-4 patients, the independent poor prognostic factors for OS, DFS, and LRFS were being male and having $\mathrm{pN}+$ disease; being male, having $\mathrm{pN}+$ disease, and sequential chemotherapy (HR, 1.781; 95\% CI, 1.086-2.921; $\mathrm{P}=0.022$ ); and $\mathrm{pN}+$ and sequential chemotherapy (HR, 2.312; 95\% CI, 1.078-4.959; $\mathrm{P}=0.031$ ), respectively.

\section{Side effects}

Acute toxicity during treatment was mild with 42 of 228 patients exhibiting grade 3 leukopenia; none had grade 3 or above skin-related toxicity, cardiac toxicity or lung toxicity (Table 2). A total of $22.4 \%$ (51/228) patients were confirmed to have varying degrees of anastomotic stenosis by endoscopy or esophagogram; 10 of them needed periodic esophageal balloon dilatation. In these 51 patients, 16 had anastomotic stenosis within half a year of surgery, which was not considered a side effect of postoperative radiotherapy.

\section{Discussion}

Esophageal cancer is the sixth most common cause of cancer-related deaths worldwide and is 3 to 4 times more common in men than in women $(1,3)$. In China, ESCC is the most common histology, and seems to be more sensitive to chemotherapy, radiation therapy, and chemoradiation than adenocarcinoma.

For resectable ESCC, neoadjuvant chemoradiotherapy followed by surgery has been regarded as a standard primary treatment and is recommended by the NCCN guidelines (4). However, a large number of patients still receive upfront surgery, which is still an option in the guidelines. For patients with R0 resection, no additional treatment other than regular follow-up is recommended by the NCCN guidelines.

Considering the poor OS rates in patients who had been treated with resection alone (7), adjuvant therapy may be necessary. Since the 1990s, postoperative radiation for esophageal cancer has been attempted. As these trials were designed more than 20 years ago by employing a 
Table 2 (Chemo)radiotherapy toxicity

\begin{tabular}{|c|c|}
\hline Toxicity & $\mathrm{n}(\%)$ \\
\hline Nausea, vomiting ( $\geq$ grade 3 ) & $8(3.5)$ \\
\hline Mucositis, dysphagia ( $\geq$ grade 3 ) & $35(15.4)$ \\
\hline Leukopenia ( $\geq$ grade 3) & $42(18.5)$ \\
\hline Skin-related toxicity ( $\geq$ grade 3 ) & 0 \\
\hline Lung toxicity ( $\geq$ grade 3 ) & 0 \\
\hline Cardiac toxicity ( $\geq$ grade 3 ) & 0 \\
\hline Toxic death ( $\geq$ grade 3 ) & 0 \\
\hline
\end{tabular}

conventional two-dimensional radiotherapy (2D-RT) technique, they failed to show a survival advantage between patients who had surgery with and without postoperative radiotherapy $(8,9)$. Since then, radiotherapy technology has developed rapidly. New technology such as fourdimensional chemotherapy, IMRT, and proton beam therapy have been applied in clinical practice, hence the role of postoperative adjuvant radiotherapy needs to be re-evaluated. In recent years, there have been many trials trying to investigate whether postoperative radiotherapy could improve cure rates compared with surgery alone, and the results seem to be affirmative (10-15).

In our retrospective study, the role of adjuvant radiotherapy in advanced ESCC patients after surgery was assessed. Most of the patients who were entered into this study had a pathologic stage II-III tumor (41.2-55.7\%) and only $7(3.1 \%)$ patients had stage IVA esophageal cancer. The median OS was 34 months with a 5 -year OS of $62.2 \%$, which was much higher than the OS of $18.6-45.8 \%$ of the surgery alone group in previous reports $(8,15-17)$.

Univariate analysis showed that patients with $\mathrm{pN}+\mathrm{had}$ a lower 5 -year OS rate (53.0\% vs. $77.8 \%, \mathrm{P}=0.000), 5$-year DFS rate $(44.1 \%$ vs. $73.4 \%, \mathrm{P}=0.000)$, and 5 -year LRFS rate $(43.8 \%$ vs. $74.2 \%, \mathrm{P}=0.000)$ than patients with $\mathrm{pN} 0$. For node-positive patients, the benefits of postoperative radiotherapy seemed to be somewhat weak; therefore, concurrent and sequential chemotherapy was attempted.

SWOG 9008/INT-0116 investigated the effectiveness of surgery plus postoperative chemoradiation on the survival of patients with resectable adenocarcinoma of the stomach or esophagogastric junction (18). For ESCC, there are no such landmark intergroup trials, but some retrospective studies have affirmed the outcome of our study.

Hsu (19) retrospectively reviewed data from 290 ESCC patients who had undergone esophagectomy. Of these patients, 104 underwent postoperative chemotherapy (cisplatin at $80 \mathrm{mg} / \mathrm{m}^{2}$ per day on day $1+$ fluorouracil at $600 \mathrm{mg} / \mathrm{m}^{2}$ per day on Days $1-4$ over a 21 -day cycle) plus radiotherapy (45-50.4 Gy) and 186 underwent postoperative radiotherapy alone. Their results showed that in $\mathrm{pN}+\mathrm{ESCC}$ patients, postoperative chemoradiotherapy was significantly more effective than radiotherapy alone at increasing the 3 -year OS rate from $14.1 \%$ to $45.8 \%$ and 3 -year DFS rate from $11.5 \%$ to $24.1 \%$. For pN0 patients, OS and DFS rates were similar in both groups. Another retrospective study included 304 node-positive ESCC patients who had undergone esophagectomy, consisting of the adjuvant chemoradiotherapy group $(n=164)$ and surgery alone group $(n=140)$. All the patients received 50 Gy of radiotherapy and the chemoradiotherapy group received postoperative chemotherapy (cisplatin at $80 \mathrm{mg} / \mathrm{m}^{2}$ on average on days $1-3$, plus paclitaxel at $135 \mathrm{mg} / \mathrm{m}^{2}$ on day 1 over a 21-day cycle). For node-positive ESCC patients, the 5 -year OS rate in the chemoradiotherapy group was significantly higher than that in the surgery group $(47.4 \%$ vs. $38.6 \%)$; the distant metastasis rate (33\% vs. $42 \%)$ and overall recurrence rate (70\% vs. $76 \%$ ) were lower (20).

CCT did not show a survival benefit in $\mathrm{pN}+$ patients in our retrospective study. However, in patients with stage III + IVA disease, we found that CCT had no significant effect on OS and LRFS, but it improved DFS in such patients. Univariate analysis showed that 5 -year DFS could be increased from $35.1 \%$ to $55.5 \%$ ( $\mathrm{P}=0.025)$. We did not observe the benefits of CCT for OS and LRFS as previously described, possibly because the chemotherapy regimen we used was not the same, but the univariate analysis still showed an increasing trend in 5-year LRFS $(65.3 \%$ vs. $78.6 \%, \mathrm{P}=0.061)$. In addition, considering that we have adopted three chemotherapy regimens, if we subdivide our cohort into specific chemotherapy regimens, the sample size of each group would be too small and the results obtained would not be credible, so we did not further analyze our results according to the specific chemotherapy regimens. Therefore, we may get more reliable results after unifying the chemotherapy regimen and further expanding the sample size.

The survival benefit of postoperative chemotherapy in ESCC was demonstrated in a multicenter prospective randomized phase III study (21). This study, which compared postoperative chemotherapy with two courses of cisplatin and fluorouracil to surgery alone, showed that postoperative chemotherapy was better able to prevent relapse in patients with stage II-IVA (M1 lymph node). 
Another meta-analysis compared the survival rates of postoperative chemotherapy with surgery alone across nine studies. The results supported postoperative chemotherapy as an independent favorable prognostic factor for ESCC, which could improve both OS and DFS (22). However, the two articles only compared postoperative chemotherapy with surgery alone, rather than with postoperative radiotherapy. The meta-analysis mentioned above also did not distinguish between postoperative radiotherapy combined with chemotherapy and postoperative chemotherapy alone. This makes us wonder whether the survival benefits of this result are from postoperative chemotherapy or postoperative radiotherapy.

Considering our study has clearly observed the survival benefit of postoperative radiotherapy, we compared postoperative radiotherapy plus chemotherapy with postoperative radiotherapy alone. The univariate analysis results showed that compared with the postoperative radiotherapy group, the OS, DFS, and LRFS of the sequential chemotherapy group were significantly reduced. Multivariate analysis showed that sequential chemotherapy was associated with decreased LRFS (HR, 2.359; 95\% CI, 1.251-4.448; $\mathrm{P}=0.008)$. Subgroup analysis further suggested that sequential chemotherapy could lead to poor DFS and LRFS, especially for patients in stage T3-4. In fact, the stage III + IVA patients accounted for $77.0 \%$ of the sequential chemotherapy group (67/87) and only $47.5 \%$ $(67 / 141)$ of the patients who did not undergo sequential chemotherapy. In addition, we did not initially assess the general condition of the patients, which was also affected by the stage of the patients, resulting in a poor prognosis in patients with sequential chemotherapy because of the higher number of patients with stage III + IVA disease and lower Karnofsky performance score (KPS) than those of the control group. Therefore, if sequential chemotherapy did not bring absolute survival benefits, it would be difficult to observe better survival in the sequential chemotherapy group than in the control group from our study. Moreover, the consistency of the chemotherapy regimens and the insufficient sample size also had an impact on the results, so this result of our study needs to be further verified.

In our study, all treatment side effects were acceptable. Although some patients had anastomotic stenosis, we were not sure whether it was caused by surgery or postoperative radiotherapy.

As we mentioned above, there were some limitations in this study. The first is that the sample size needs to be expanded; especially when subgroup analysis is performed, the sample size of each group would be even lower. In addition, we did not consider the KPS at the time of treatment and some patients may have a poor prognosis due to a lower KPS during chemotherapy. Furthermore, the chemotherapy regimens in this article were not the same and we have yet to analyze the effects of each specific chemotherapy regimen. These can only be analyzed further with an expanded sample size.

\section{Conclusions}

For locally advanced ESCC, optimal treatment guidelines for the administration of postoperative adjuvant therapy have not been established. Our study clearly observed the benefits of postoperative concurrent chemoradiotherapy on DFS in patients with stage III + IVA disease. Sequential chemoradiotherapy may be associated with a poor LRFS. In addition, being male, having a higher $\mathrm{T}$ stage, and being node-positive were considered to be independent poor prognostic factors for OS and DFS.

\section{Acknowledgments}

None.

\section{Footnote}

Conflicts of Interest: The authors have no conflicts of interest to declare.

Ethical Statement: The authors are accountable for all aspects of the work in ensuring that questions related to the accuracy or integrity of any part of the work are appropriately investigated and resolved. The study had been approved by the institutional review board (IRB) (the number of approval document: 2019-SR-410). The patient data in this study were retrieved from our hospital medical record system, the informed consent form was waived. The study outcomes will not affect the future management of the patients.

\section{References}

1. Bray F, Ferlay J, Soerjomataram I, et al. Global cancer statistics 2018: GLOBOCAN estimates of incidence and mortality worldwide for 36 cancers in 185 countries. CA Cancer J Clin 2018;68:394-424.

2. Chen W, Sun K, Zheng R, et al. Cancer incidence and 
mortality in China, 2014. Chin J Cancer Res 2018;30:1-12.

3. Siegel RL, Miller KD, Jemal A. Cancer statistics, 2019. CA Cancer J Clin 2019;69:7-34.

4. Ajani JA, D'Amico TA, Bentrem DJ, et al. Esophageal and esophagogastric junction cancers, version 2.2019, NCCN clinical practice guidelines in oncology. J Natl Compr Canc Netw 2019;17:855-83.

5. Wong AT, Shao M, Rineer J, et al. The impact of adjuvant postoperative radiation therapy and chemotherapy on survival after esophagectomy for esophageal carcinoma. Ann Surg 2017;265:1146-51.

6. Schreiber D, Rineer J, Vongtama D, et al. Impact of postoperative radiation after esophagectomy for esophageal cancer. J Thorac Oncol 2010;5:244-50.

7. Kleinberg L, Forastiere AA. Chemoradiation in the management of esophageal cancer. J Clin Oncol 2007;25:4110-7.

8. Ténière P, Hay JM, Fingerhut A, et al. Postoperative radiation therapy does not increase survival after curative resection for squamous cell carcinoma of the middle and lower esophagus as shown by a multicenter controlled trial. French University Association for Surgical Research. Surg Gynecol Obstet 1991;173:123-30.

9. Zieren HU, Müller JM, Jacobi CA, et al. Adjuvant postoperative radiation therapy after curative resection of squamous cell carcinoma of the thoracic esophagus: a prospective randomized study. World J Surg 1995;19:444-9.

10. Zhang W, Liu X, Xiao Z, et al. Postoperative intensitymodulated radiotherapy improved survival in lymph nodepositive or stage III thoracic esophageal squamous cell carcinoma. Oncol Res Treat 2015;38:97-102.

11. Chen SB, Weng HR, Wang G, et al. The impact of adjuvant radiotherapy on radically resected $\mathrm{T} 3$ esophageal squamous cell carcinoma. J Cancer Res Clin Oncol 2016;142:277-86.

12. Liu T, Liu W, Zhang H, et al. The role of postoperative radiotherapy for radically resected esophageal squamous cell carcinoma: a systemic review and meta-analysis. J Thorac Dis 2018;10:4403-12.

Cite this article as: Zhang Z, Xu L, Di X, Zhang C, Ge X, Sun $\mathrm{X}$. A retrospective study of postoperative radiotherapy for locally advanced esophageal squamous cell carcinoma. Ann Palliat Med 2019;8(5):708-716. doi: 10.21037/apm.2019.11.19
13. Zhao XH, Wang D, Wang F, et al. Comparison of the effect of postoperative radiotherapy with surgery alone for esophagus squamous cell carcinoma patients: a metaanalysis. Medicine (Baltimore) 2018;97:e13168.

14. Yeh HL, Hsu CP, Lin JC, et al. A retrospective study of postoperative chemoradiotherapy for locally advanced esophageal squamous cell carcinoma. Formos J Surg 2012;45:172-7.

15. Xiao ZF, Yang ZY, Liang J, et al. Value of radiotherapy after radical surgery for esophageal carcinoma: a report of 495 patients. Ann Thorac Surg 2003;75:331-6.

16. Tachibana M, Kinugasa S, Yoshimura H, et al. Clinical outcomes of extended esophagectomy with three-field lymph node dissection for esophageal squamous cell carcinoma. Am J Surg 2005;189:98-109.

17. Shapiro J, van Lanschot JJB, Hulshof MCCM, et al. Neoadjuvant chemoradiotherapy plus surgery versus surgery alone for oesophageal or junctional cancer (CROSS): long-term results of a randomised controlled trial. Lancet Oncol 2015;16:1090-8.

18. Macdonald JS, Smalley SR, Benedetti J, et al. Chemoradiotherapy after surgery compared with surgery alone for adenocarcinoma of the stomach or gastroesophageal junction. N Engl J Med 2001;345:725-30.

19. Hsu PK, Huang CS, Wang BY, et al. Survival benefits of postoperative chemoradiation for lymph node-positive esophageal squamous cell carcinoma. Ann Thorac Surg 2014;97:1734-41.

20. Chen J, Pan J, Liu J, et al. Postoperative radiation therapy with or without concurrent chemotherapy for nodepositive thoracic esophageal squamous cell carcinoma. Int J Radiat Oncol Biol Phys 2013;86:671-7.

21. Ando N, Iizuka T, Ide H, et al. Surgery plus chemotherapy compared with surgery alone for localized squamous cell carcinoma of the thoracic esophagus: a Japan Clinical Oncology Group Study--JCOG9204. J Clin Oncol 2003;21:4592-6.

22. Zhao P, Yan W, Fu H, et al. Efficacy of postoperative adjuvant chemotherapy for esophageal squamous cell carcinoma: a meta-analysis. Thorac Cancer 2018;9:1048-55. 